\title{
Effects of Temperature and Culture Media Composition on Sporulation, Mycelial Growth, and Antifungal Activity of Isaria javanica pf185
}

*Corresponding author

Tel: $+82-62-530-2071$

Fax: +82-62-530-0208

E-mail: yckimyc@jnu.ac.kr ORCID

https://orcid.org/0000-0002-7661-7600

Received July 16, 2021

Revised September 13, 2021

Accepted September 14, 2021

\author{
Jang Hoon Lee', Yong Seong Lee ${ }^{2}$, and Young Cheol Kim ${ }^{3} * \llbracket$ \\ 'BASF Korea Ltd., Agricultural Solutions, Seoul 04518, Korea \\ ${ }^{2}$ Department of Research and Development, Agricultural Technology Center, Sunchang 56025, Korea \\ ${ }^{3}$ Department of Applied Biology, College of Agriculture and Life Sciences, Chonnam National University, \\ Gwangju 61186, Korea
}

\begin{abstract}
The fungal isolate Isaria javanica pf185 has potential as a mycopesticide because it demonstrates insecticidal activity against the green peach aphid and antifungal activity against Colletotrichum gloeosporioides. For commercialization of this isolate, determination of the optimal and least expensive culture conditions is required; however, these data are not currently available. This study describes the conditions for optimal development of conidia and production of metabolites for the biocontrol of the fungal pathogen. The optimal culture conditions were examined using cultures on solid agar and liquid media. High growth temperature enhanced spore formation but reduced antifungal activity in both solid and liquid media. The highest spore yield was obtained in a medium containing glucose as a carbon source and yeast extract as a nitrogen source. Soybean powder and wheat bran were effective nitrogen sources that promoted spore production and antifungal activity of the isolate. These results revealed the basic, cost-effective growth media for commercial production of a biopesticide with insecticidal and antifungal properties for use in integrated pest management.
\end{abstract}

Keywords: Antifungal activity, Growth temperature, Mycopesticide, Nitrogen sources

\section{Introduction}

The development of biopesticides to minimize or replace chemical pesticides is an active field of research with value to sustainable and organic agriculture. Several insect pathogenic fungi are plant endophytes that enhance plant growth and reduce plant microbial diseases (Jaber and Ownley, 2018). Entomopathogenic fungi have already been commercialized for various purposes (de Faria and Wraight, 2007; Lacey et al., 2015; Sandhu et al., 2012). Isaria sp. has insecti-

Research in Plant Disease

pISSN 1598-2262, elSSN 2233-9191

www.online-rpd.org cidal activity against the sweet potato whitefly Bemisia tabaci (Cabanillas and Jones, 2009b; Faria and Wraight, 2001), and can control the Asian citrus psyllid, aphids, and arboreal termites (Cabanillas and Jones, 2009b; da Silva Lopes et al., 2017; Hunter et al., 2011). Moreover, Isaria isolates inhibit fungal plant pathogens (Kang et al., 2018), and in cabbage, they promote plant growth even under water-stress conditions (Dara et al., 2017).

To be successful in the biopesticide market, formulations of entomopathogenic fungi must have high spore levels and an effective antagonistic potential. High conidial numbers have a positive impact on mycoparasitism (Jackson et al., 2010; Sandhu et al., 2012). Both liquid and solid growth media are used for culturing entomopathogenic fungi (Burges, 
1998). Conidial formation is influenced by the types of carbon $(\mathrm{C})$ and nitrogen $(\mathrm{N})$ sources and the $\mathrm{C}: \mathrm{N}$ ratio (Gao and Liu, 2010; Gao et al., 2007). The isolate Isaria javanica pf04 was successfully cultured for virulence against second-instar whitefly nymphs using single-phase, solid-state fermentation on a barley substrate supplemented with additives that included organic silkworm powder, moth larvae, and inorganic $\mathrm{CaCO}_{3}$ or $\mathrm{CaSO}_{4}$ (Kim et al., 2014; Xie et al., 2016). Growth temperature was a key factor determining mycelial mass and spore production for I. fumosoroseus (Fargues and Bon, 2004). The optimal growth temperature for many Isaria sp. isolates is around $25^{\circ} \mathrm{C}$, with no growth occurring above $35^{\circ} \mathrm{C}$ (Fargues and Bon, 2004; Vidal et al., 1997; Xie et al., 2016).

The isolate I. javanica pf185 displays dual biocontrol: it acts as an insecticide against the green peach aphid and as a mycocide against the fungal pathogen, a causative agent of anthracnose in red pepper (Kang et al., 2018). This dual property is attributed to dibutyl succinate, a metabolite produced by the isolate, which has both insecticidal and antifungal activities (Lee et al., 2019). In the present study, culture conditions were examined by varying the growth medium composition and temperature to determine the optimal mycelial mass, spore formation, and antifungal activity. Our findings provide insight into the liquid and solid culture conditions that maximize conidial production and the antifungal activity of I.javanica pf 185.

\section{Materials and Methods}

Fungal strains and growth maintenance. I.javanica pf185 was isolated from Korean mountain soil; the isolate was demonstrated to function as an insect pathogen capable of killing the larvae of the greater wax moth Galleria mellonella (Kang et al., 2018). The isolate was deposited in the Korean Agricultural Culture Collection (KACC) at the National Agrobiodiversity Center, Wanju, South Korea, with the designation KACC 93241P. Colletotrichum gloeosporioides, a plant pathogen that causes anthracnose on vegetables, obtained as isolate KACC 40896, was used in tests of antifungal activity. All fungal isolates were grown and maintained on potato dextrose agar (PDA; Difco Inc., Detroit, MI, USA). Stock cultures were stored at $-80^{\circ} \mathrm{C}$ as $1 \mathrm{~mm}^{2}$ discs (plugs) of established PDA agar cultures immersed in 10\% sterile glycerol. Cultures were established by transferring the stock plug to PDA plates and incubating at $25^{\circ} \mathrm{C}$ for 7 days for both mycelial growth and conidia formation. When needed, conidia were harvested as water suspensions by flooding the plate surface and filtering through two layers of sterile cheese cloth to remove hyphal debris.

Culture condition of I. javanica pf185. To determine the effect of culture temperature on mycelial mass, spore formation, and antifungal activity of I. javanica pf 185 in the solid culture condition, the $7 \mathrm{~mm}^{2}$ mycelial plugs of I. javanica pf185 were placed in the center of Sabouraud dextrose agar plates supplemented with $1 \%$ yeast extract (SDYA; pH 6.5, Difco Inc.), one per plate, and incubated at $20.0^{\circ} \mathrm{C}, 22.5^{\circ} \mathrm{C}$, $25.0^{\circ} \mathrm{C}, 27.5^{\circ} \mathrm{C}$, and $30.0^{\circ} \mathrm{C}$ in the dark for 7 days. For liquid culture, the $100 \mathrm{ml}$ of Sabouraud dextrose yeast extract liquid medium was sterilized in $250 \mathrm{ml}$ baffled Erlenmeyer flasks. I. javanica pf185 was inoculated at 100 conidia/ml and incubated for 7 days at $25^{\circ} \mathrm{C}, 25^{\circ} \mathrm{C}$, and $30^{\circ} \mathrm{C}$ with shaking at $150 \mathrm{rpm}$ on a rotary shaker.

Basal salts medium with a C:N ratio of 10:1 was prepared by using a 3\% C and a $0.3 \% \mathrm{~N}$ source, and then used to examine the effects of different $C$ and $N$ sources on the mycelial growth, spore formation, and antifungal activity of I.javanica pf185. The basal salts medium contained the following: 0.3 $\mathrm{g} / \mathrm{KH}_{2} \mathrm{PO}_{4}, 0.3 \mathrm{~g} / \mathrm{l} \mathrm{K} \mathrm{HPO}_{4}$, and $0.3 \mathrm{~g} / \mathrm{l} \mathrm{MgSO}{ }_{4}$, with a final $\mathrm{pH}$ of 6.5. The $\mathrm{C}$ sources used were glucose, mannitol, and molasses (EM Green Inc., Seoul, Korea). The N sources were yeast extract (Difco Inc.), wheat bran (Korea Wheat Farming Association Corp., Gurye, Korea), soybean powder (Hansalim Co., Gwangju, Korea), and urea (Sigma-Aldrich Company, St. Louis, MO, USA). Solid media were prepared with $1.5 \%$ agar. For liquid culture, l.javanica pf185 (100 conidia/ml) was inoculated on the $100 \mathrm{ml}$ of basal salts medium (C:N ratio of 10:1) and incubated for 7 days at $25^{\circ} \mathrm{C}$ with shaking at $150 \mathrm{rpm}$ on a rotary shaker.

Estimation of mycelial growth, spore formation, and antifungal activity. For solid culture, the average diameter of the fungal colony of I. javanica pf185 was measured in four different directions using a digital ruler (Mitutovo Corp., Kanagawa, Japan). To determine spore formation of $I$. javanica pf185, four $0.5 \mathrm{~cm}^{2}$ agar plugs were excised from the edge of the culture using sterile razor blades and transferred to $1 \mathrm{ml}$ of sterile water in Eppendorf tubes before vigorous vortexing for $10 \mathrm{~min}$. The spores in the suspension were counted using a hemocytometer (Marienfeld Superior, Lau- 
da-Königshofen, Germany) under a light microscope (Leica M165 FC, Leica Microsystems, Wetzlar, Germany). Antifungal activity was assessed by co-culturing I.javanica pf185 and the test pathogenic fungus $C$. gloeosporioides. Mycelial plugs of I. javanica pf185 and C. gloeosporioides grown on PDA were placed $5 \mathrm{~cm}$ apart on the SDYA or basal salts agar medium (C:N ratio of 10:1). Plates inoculated with only one of the two fungi were used as controls. After growth for 7 days at $25^{\circ} \mathrm{C}$, mycelial growth was quantified as the average diameter of the colony size in four different directions for each of the growth conditions. Antifungal activity was calculated using the formula: $[(A-B) / A] \times 100$, where $A$ is growth of the fungi with no interactions (controls) and $B$ is the growth in the coculture.

For liquid culture, each culture broth $(100 \mathrm{ml})$ was filtered through two layers of sterile cheesecloth to separate mycelia and spores. The mycelium was then washed three times with distilled water to remove the liquid medium, and the dry mass was measured by weighing after drying at $65^{\circ} \mathrm{C}$ for 2 days. The remained filtrate (spore suspension) was serially
A Solid medium

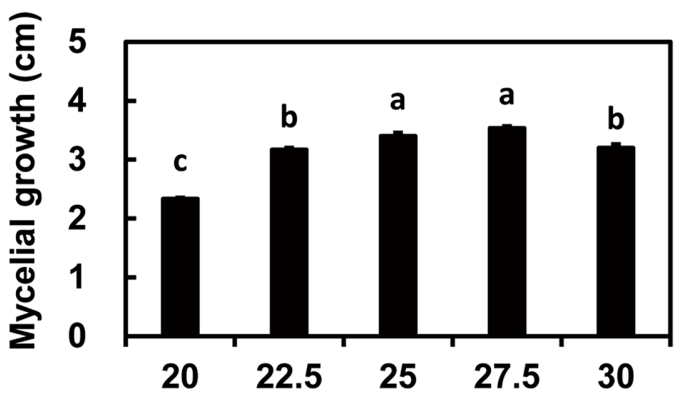

B

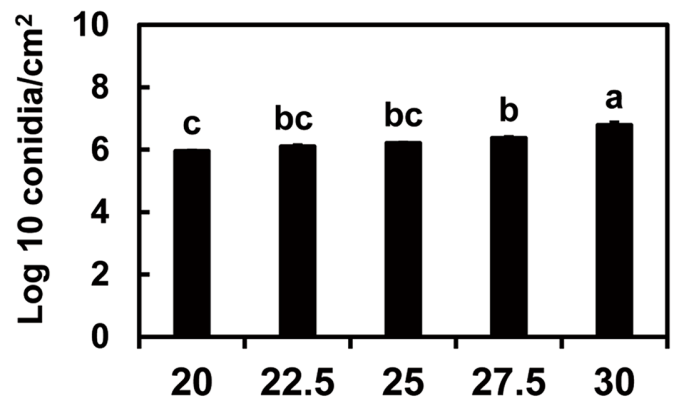

C

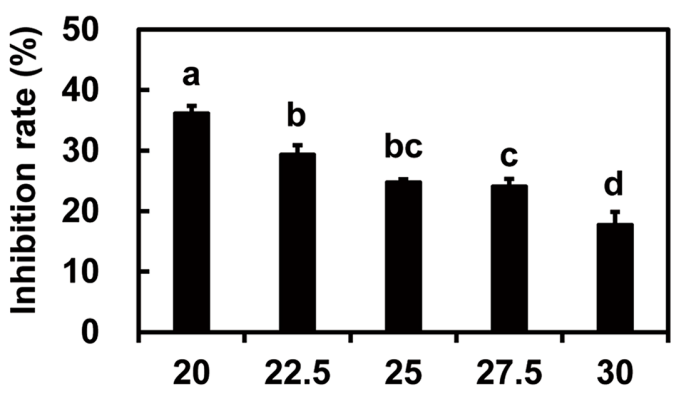

Liquid medium
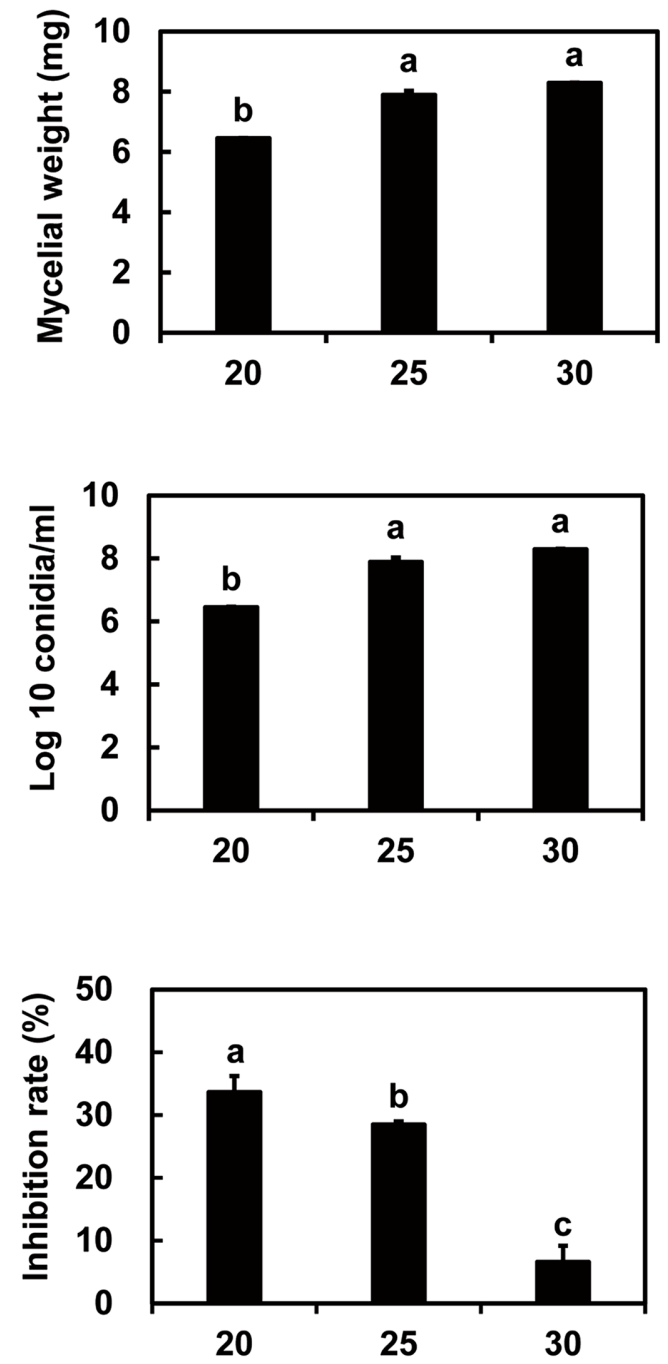

Growth temperature $\left({ }^{\circ} \mathrm{C}\right)$

Fig. 1. Effects of temperature on fungal mycelia (A), spore formation (B), and antifungal activity (C) of Isaria javanica pf185 grown on Sabouraud dextrose yeast extract medium. Culture temperature ranged from $20-30^{\circ} \mathrm{C}$. Data are presented as the mean \pm standard deviation of two independent experiments with three replicates per treatment. Data were analyzed using one-way ANOVA $(P<0.05)$; if the $F$ test was significant, the differences were further compared using the Duncan's multiple range test. Different letters indicate significant differences at $P<0.05$. 
diluted and the number of spores was determined using a hemocytometer. The cell-free culture filtrate obtained by filtering through a sterile $0.2-\mu \mathrm{m}$ filter (Millipore Filter Corp., Bedford, MA, USA) was mixed in a 1:1 (v/v) ratio with sterilized melted PDA medium containing 3\% agar and poured into petri dishes. After solidification, the $7 \mathrm{~mm}^{2}$ mycelial plug of $C$. gloeosporioides grown for 7 days on PDA was placed in the center of the assay plates. All experiments were repeated at least twice with three replicates per treatment.

Statistical analysis. Data were analyzed using one-way analysis of variance (ANOVA; $P<0.05$ was considered significant), with the IBM SPSS software version 23 (IBM Corp., Ar-

A

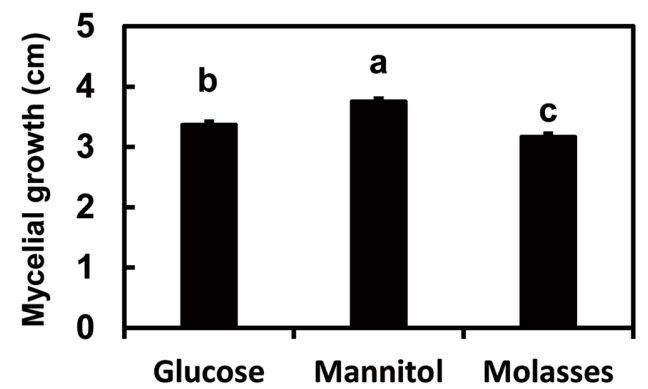

B

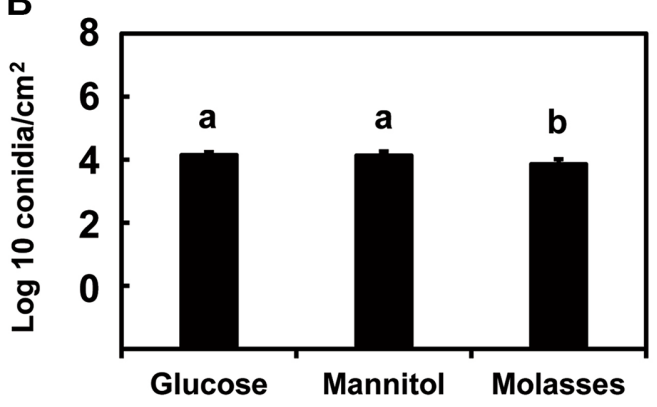

C

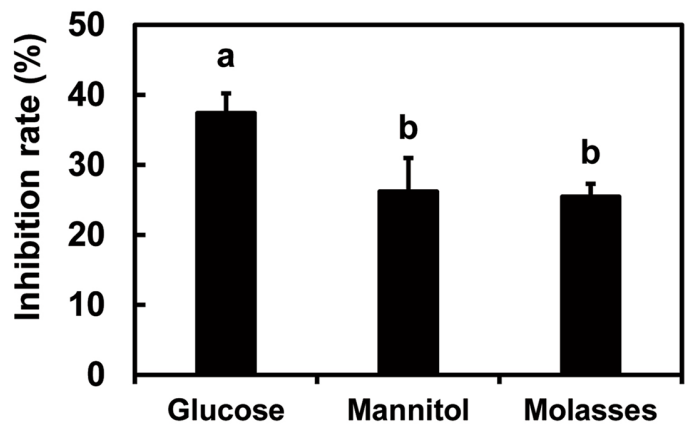

monk, NY, USA); if the $F$ test was significant, differences were further compared using Duncan's multiple range test $(P<$ 0.05).

\section{Results}

Growth and antifungal activity of $I$. javanica pf 185 on SDYA. The effects of temperature on the growth and antifungal activity were investigated in SDYA, which has a low C:N ratio because of the presence of casein and yeast extract as $\mathrm{N}$ sources. No growth of I. javanica was observed at or above $35^{\circ} \mathrm{C}$, as anticipated from previous findings (data not shown). The effects of growth between $20^{\circ} \mathrm{C}$ and $30^{\circ} \mathrm{C}$ are presented
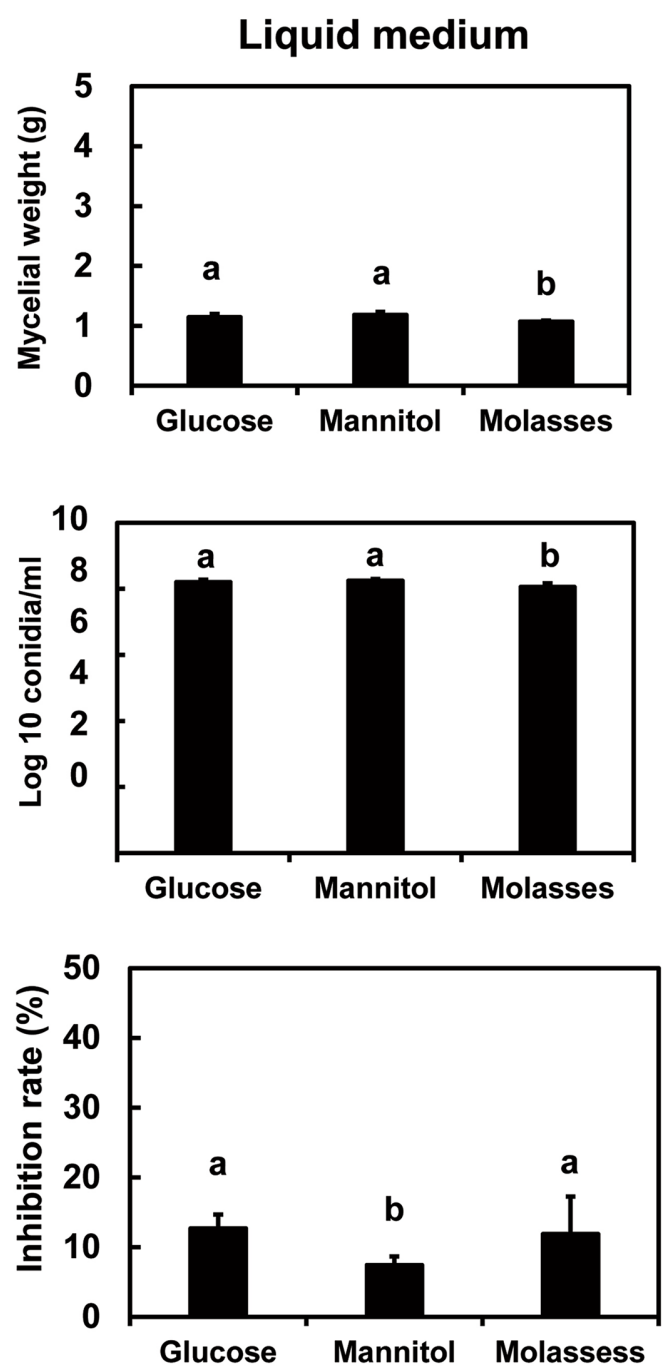

Carbon source

Fig. 2. Effects of different carbon sources on mycelial growth (A), spore formation (B), and antifungal activity (C) of Isaria javanica pf185. Studies were performed with solid and liquid basal salts media containing $3 \%$ carbon source (glucose, mannitol, or molasses) and $0.3 \%$ yeast extract. Data are presented as the mean \pm standard deviation of two independent experiments with three replicates per treatment. Different letters indicate significant differences by Duncan's multiple range test $(P<0.05)$. 
in Fig. 1. Mycelial growth on solid medium was maximal between $25^{\circ} \mathrm{C}$ and $27.5^{\circ} \mathrm{C}$ (Fig. 1A), whereas spore formation was maximal at $30^{\circ} \mathrm{C}$ (Fig. 1B). However, the antifungal activity due to the secreted metabolites declined with increasing temperature from $20^{\circ} \mathrm{C}$ to $30^{\circ} \mathrm{C}$ (Fig. 1C). Thus, growth at $20^{\circ} \mathrm{C}$ was optimal for antifungal activity, but growth and spore production were favored at $30^{\circ} \mathrm{C}$.

Effect of different $\mathrm{C}$ and $\mathrm{N}$ sources on the mycelial growth and spore formation of $I_{\text {. javanica }}$ pf 185 . To understand the effects of $\mathrm{C}$ and $\mathrm{N}$ sources in the culture medium, basal salts media were used with a C:N ratio of 10:1 and yeast extract as the $\mathrm{N}$ source. Mycelial growth, measured as colony diameters on solid media or as dry masses in liquid cultures, is shown in Fig. 2A and sporulation in Fig. 2B. Fungal spore production was lower in the basal salts agar medium than in SDYA, but higher than that in the liquid culture. In the basal salts media with yeast extract, mycelial mass and spore formation were slightly but significantly higher on glucose or mannitol than on molasses (Fig. 2A, B).
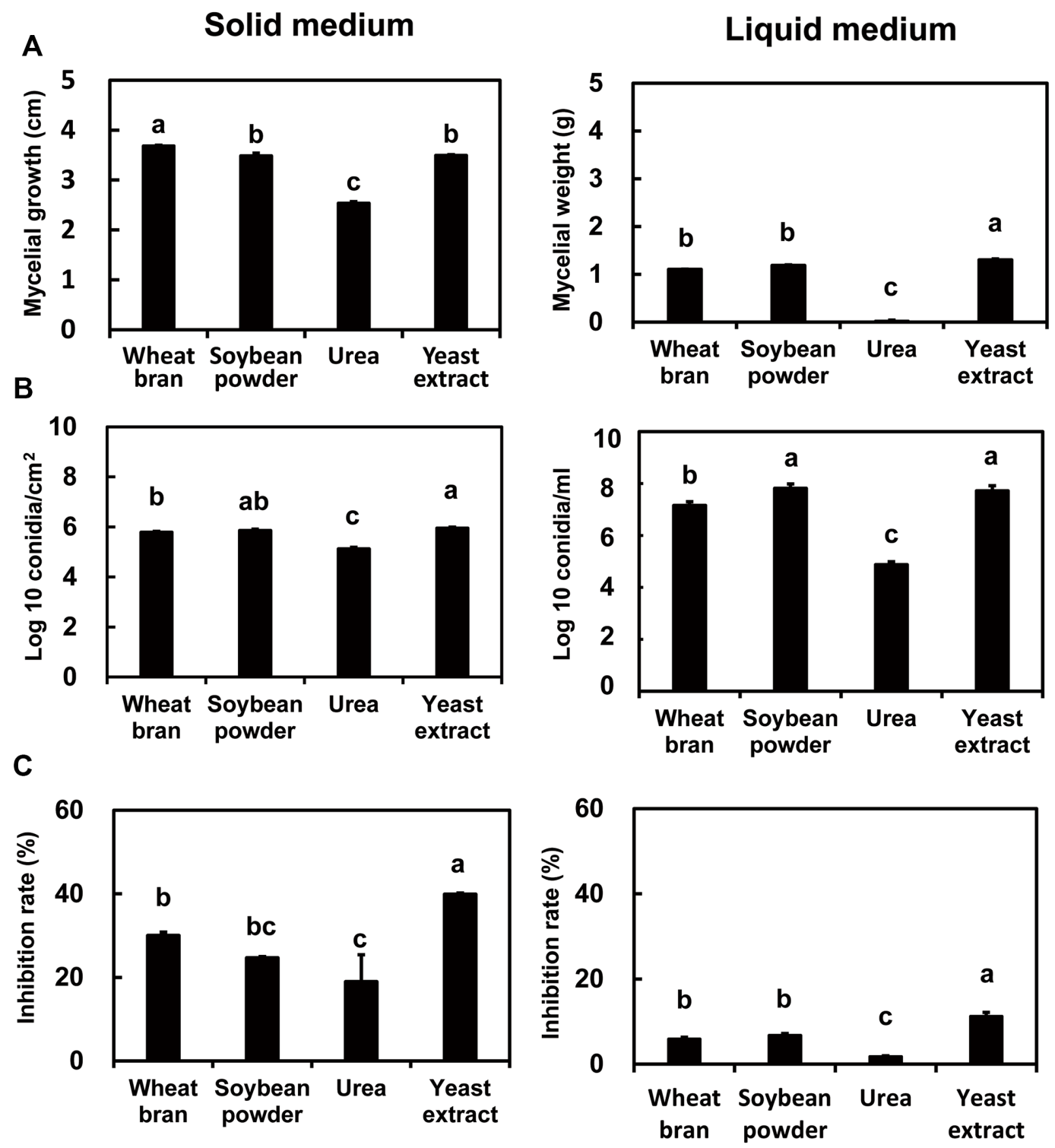

Nitrogen source

Fig. 3. Effects of different nitrogen sources on mycelial growth (A), spore formation (B), and antifungal activity (C) of Isaria javanica pf185. Studies were performed with solid and liquid basal salts media containing $3 \%$ glucose and $0.3 \%$ nitrogen source (wheat bran, soybean powder, or urea). Data are presented as the mean \pm standard deviation of two independent experiments with three replicates per treatment. Different letters indicate significant differences by Duncan's multiple range test $(P<0.05)$. 
The $\mathrm{N}$ source in the basal salts medium at a C:N ratio of 10:1 with glucose as the $C$ source also affected growth on both the solid and liquid media. Urea was the least productive $N$ source for mycelial growth and spore formation, possibly because the other $\mathrm{N}$ sources had additional growth-boosting components (Fig. 2C, D). Mycelial growth of isolate pf185 was significantly higher with wheat bran powder in the solid medium and yeast extract in the liquid medium as $\mathrm{N}$ sources (Fig. 2C). Supplementation with yeast extract resulted in significantly higher spore formation in both the liquid and solid media (Fig. 2D).

Effect of $\mathrm{C}$ and $\mathrm{N}$ sources on the antifungal activity of I. javanica pf185. The isolate pf185 secreted metabolites that inhibited the growth of the pathogen fungus C. gloeosporioides on all media tested. On the solid basal salts medium with a 10:1 C:N ratio and yeast extract as the $\mathrm{N}$ source, inhibition was greater with glucose as the $\mathrm{C}$ source than with either mannitol or molasses. However, in the liquid medium, glucose and molasses were better $C$ sources for antifungal activity (Fig. 3A). In both liquid and solid media, antifungal activity of isolate pf 185 grown with yeast extract as the $N$ source was significantly higher than with other $\mathrm{N}$ sources (Fig. 3B). In addition, the low growth potential in the liquid and solid media with urea correlated with significantly reduced inhibition of $C$. gloeosporioides (Fig. 3B).

\section{Discussion}

This study established that media containing glucose and yeast extract supported mycelial growth, sporulation, and the secretion of antifungal metabolites by I. javanica pf 185 . When cultured on the amended Sabouraud medium, mycelial mass and spore formation were optimal between 20 and $30^{\circ} \mathrm{C}$. However, secreted antifungal metabolites from pf185 showed highest antifungal activity against the fungus causing anthracnose, $\mathrm{C}$. gloeosporioides, at $20^{\circ} \mathrm{C}$. In previous studies with other Isaria spp., strain variability in sporulation with medium composition was noted (Ali et al., 2009, 2010; Gao and Liu, 2010; Gao et al., 2007). Three isolates of I. fumosoroseus had highest spore formation on a medium with the same C:N ratio (10:1) as that used in our study (Ali et al., 2009; Jackson et al., 1997; Vega et al., 2003). Sporulation of Paecilomyces lilacinus was maximal at a C:N ratio of 10:1 for one isolate but a higher C:N ratio was needed for others (Gao et al., 2007). Our experiments with the basal salts medium show that glucose was an effective $C$ source compared to the costlier mannitol and molasses. For the $\mathrm{N}$ source, wheat bran and soybean powder, but not urea, could be substituted for yeast extract in the development of an inexpensive culture medium for commercial purposes. These results for $l$. javanica pf185 indicate a flexibility in medium composition while maintaining spore formation.

Antifungal activity from the isolate was also influenced by $\mathrm{C}$ and $\mathrm{N}$ sources by as yet unknown mechanisms. Present evidence shows that dibutyl succinate is a metabolite with both antifungal and aphicidal activities (Lee et al., 2019); studies that clarify how nutrition affects its production in pf185 are in progress. Metabolic analysis of mycelia and spores of another insect pathogenic fungus, Beauveria bassiana, implicated a decrease in primary metabolism and an increase in secondary metabolism as ways to protect spores against harmful stresses (Liu et al., 2015). With the biocontrol agent Talaromyces flavus, $\mathrm{C}$ and $\mathrm{N}$ sources in a defined liquid salt medium increased ascospore production, but reduced biocontrol potential against Verticillium wilt disease when compared with growth on PDA (Engelkes et al., 1997).

Our finding that temperatures above $20^{\circ} \mathrm{C}$ reduced antifungal activity indicated that cultivation at a lower temperature rather than at the temperature optimal for fungal growth, near $30^{\circ} \mathrm{C}$, was optimal. The effects of temperature on the fungal mass of this Isaria isolate were similar to other findings for Isaria and Paecilomyces (Cabanillas and Jones, 2009a; Fargues and Bon, 2004; Vidal et al., 1997). Isaria isolates, such as I. fumosorosea, are generally mesophiles (Vidal et al., 1997) although some are more thermotolerant, such as isolates from Texas (Cabanillas and Jones, 2009a). The deviation between growth and antifungal activity with increasing temperature is a novel finding very pertinent for the growth conditions in commercial production of a biocontrol agent.

Other materials may be added to the growth media to improve the virulence of entomopathogenic fungi. The addition of chitin to liquid growth medium for I. fumosorosea isolates induced the production of insect cuticle-degrading enzymes, enhancing biocontrol of the diamondback moth (Ali et al., 2010). Palm kernel oil was a cost-effective substrate promoting spore production for I. javanica and B. bassiana, and when coupled with high humidity, the formulations caused high mortality of whitefly (do Nascimento Silva et al., 2018). Additions of $\mathrm{CaCO}_{3}, \mathrm{CaSO}_{4}$, and powders of silkworm 
and moth larvae to cultures of I. javanica pf05 enhanced spore production to promote virulence against whitefly (Kim et al., 2014; Xie et al., 2016).

In summary, the choice of nutrients and their composition as well as temperature and growth on solid or liquid media are important variables that must be considered for commercialization of I. javanica pf185 as a biocontrol agent. Our results indicated that minimal medium containing glucose and yeast extract, wheat bran, or soybean powder for inexpensive growth medium supported growth with maximum sporulation and secretion of antifungal metabolites from the isolate I.javanica pf185.

\section{Conflicts of Interest}

No potential conflicts of interest relevant to this article was reported.

\section{Acknowledgments}

This work was supported by Korea Institute of Planning and Evaluation for Technology in Food, Agriculture and Forestry (IPET) through Agricultural Machinery/Equipment Localization Technology Development Program, funded by Ministry of Agriculture, Food and Rural Affairs (MAFRA) (321055-05).

\section{References}

Ali, S., Huang, Z. and Ren, S. 2009. Media composition influences on growth, enzyme activity, and virulence of the entomopathogen hyphomycete Isaria fumosoroseus. Entomol. Exp. Appl. 131: 3038.

Ali, S., Huang, Z. and Ren, S. 2010. Production of cuticle degrading enzymes by Isaria fumosorosea and their evaluation as a biocontrol agent against diamondback moth. J. Pest Sci. 83: 361370.

Burges, H. D. 1998. Formulation of mycoinsecticides. In: Formulation of Microbial Biopesticides: Beneficial Microorganisms, Nematodes and Seed Treatments, ed. by H. D. Burges, pp. 131185. Springer, Dordrecht, The Netherlands.

Cabanillas, H. E. and Jones, W. A. 2009a. Effects of temperature and culture media on vegetative growth of an entomopathogenic fungus Isaria sp. (Hypocreales: Clavicipitaceae) naturally affecting the whitefly, Bemisia tabaci in Texas. Mycopathologia 167: 263-271.

Cabanillas, H. E. and Jones, W. A. 2009b. Pathogenicity of Isaria sp. (Hypocreales: Clavicipitaceae) against the sweet potato whitefly B biotype, Bemisia tabaci (Hemiptera: Aleyrodidae). Crop
Prot. 28: 333-337.

Dara, S. K., Dara, S. S. R. and Dara, S. S. 2017. Impact of entomopathogenic fungi on the growth, development, and health of cabbage growing under water stress. Am. J. Plant Sci. 8: 12241233.

de Faria, M. R. and Wraight, S. P. 2007. Mycoinsecticides and Mycoacaricides: a comprehensive list with worldwide coverage and international classification of formulation types. Biol. Control 43: 237-256.

da Silva Lopes, R., de Lima, G., dos Santos Correia, M. T., da Costa, A. F., de Luna Alves Lima, E. and de Menezes Lima, V. L. 2017. The potential of Isaria spp. as a bioinsecticide for the biological control of Nasutitermes corniger. Biocontrol Sci. Technol. 27: 1038-1048.

do Nascimento Silva, J., Mascarin, G. M., dos Santos Gomes, I. C., Tinôco, R. S., Quintela, E. D., dos Reis Castilho, L. et al. 2018. New cost-effective bioconversion process of palm kernel cake into bioinsecticides based on Beauveria bassiana and Isaria javanica. Appl. Mlcrobiol. Biotechnol. 102: 2595-2606.

Engelkes, C. A., Nuclo, R. L. and Fravel, D. R. 1997. Effect of carbon, nitrogen, and $\mathrm{C}: \mathrm{N}$ ratio on growth, sporulation, and biocontrol efficacy of Talaromyces flavus. Phytopathology 87: 500-505.

Fargues, J. and Bon, M. C. 2004. Influence of temperature preferences of two Paecilomyces fumosoroseus lineages on their coinfection pattern. J. Invertebr. Pathol. 87: 94-104.

Faria, M. and Wraight, S. P. 2001. Biological control of Bemisia tabaci with fungi. Crop Prot. 20: 767-778.

Gao, L. and Liu, X. 2010. Effects of carbon concentrations and carbon to nitrogen ratios on sporulation of two biological control fungi as determined by different culture methods. Mycopathologia 169: 475-481.

Gao, L., Sun, M. H., Liu, X. Z. and Che, Y. S. 2007. Effects of carbon concentration and carbon to nitrogen ratio on the growth and sporulation of several biocontrol fungi. Mycol. Res. 111: 87-92.

Hunter, W. B., Avery, P. B., Pick, D. and Powell, C. A. 2011. Broad spectrum potential of Isaria fumosorosea against insect pests of citrus. Fla. Entomol. 94: 1051-1054.

Jaber, L. R. and Ownley, B. H. 2018. Can we use entomopathogenic fungi as endophytes for dual biological control of insect pests and plant pathogens? Biol. Control 116: 36-45.

Jackson, M. A., Dunlap, C. A. and Jaronski, S. T. 2010. Ecological considerations in producing and formulating fungal entomopathogens for use in insect biocontrol. BioControl 55: 129-145.

Jackson, M. A., McGuire, M. R., Lacey, L. A. and Wraight, S. P. 1997. Liquid culture production of desiccation tolerant blastospores of the bioinsecticidal fungus Paecilomyces fumosoroseus. Mycol. Res. 101: 35-41.

Kang, B. R., Han, J. H., Kim, J. J. and Kim, Y. C. 2018. Dual biocontrol potential of the entomopathogenic fungus, Isaria javanica, for both aphids and plant fungal pathogens. Mycobiology 46: 440447.

Kim, J. J., Xie, L., Han, J. H. and Lee, S. Y. 2014. Influence of additives on the yield and pathogenicity of conidia produced by solid 
state cultivation of an Isaria javanica isolate. Mycobiology 42: 346-352.

Lacey, L. A., Grzywacz, D., Shapiro-llan, D. I., Frutos, R., Brownbridge, M. and Goettel, M. S. 2015. Insect pathogens as biological control agents: back to the future. J. Invertebr. Pathol. 132: 1-41.

Lee, Y.-S., Han, J. H., Kang, B. R. and Kim, Y. C. 2019. Dibutyl succinate, produced by an insect-pathogenic fungus, Isaria javanica pf185, is a metabolite that controls of aphids and a fungal disease, anthracnose. Pest. Manag. Sci. 75: 852-858.

Liu, H., Zhao, X., Guo, M., Liu, H. and Zheng, Z. 2015. Growth and metabolism of Beauveria bassiana spores and mycelia. BMC Microbiol. 15: 267.

Sandhu, S. S., Sharma, A. K., Beniwal, V., Goel, G., Batra, P., Kumar, A. et al. 2012. Myco-biocontrol of insect pests: factors involved, mechanism, and regulation. J. Pathog. 2012: 126819.

Vega, F. E., Jackson, M. A., Mercadier, G. and Poprawski, T. J. 2003. The impact of nutrition on spore yields for various fungal entomopathogens in liquid culture. World J. Microbiol. Biotechnol. 19: 363-368.

Vidal, C., Fargues, J. and Lacey, L. A. 1997. Intraspecific variability of Paecilomyces fumosoroseus: effect of temperature on vegetative growth. J. Invertebr. Pathol. 70: 18-26.

Xie, L., Han, J. H., Kim, J. J. and Lee, S. Y. 2016. Effects of culture conditions on conidial production of the sweet potato whitefly pathogenic fungus Isaria javanica. Mycoscience 57: 64-70. 